\title{
Bassin de Korçë, Kallamas
}

Petrika Lera, Gilles Touchais, Cécile Oberweiler, Ole Christian Aslaksen, Charlotte Blein, Athina Boleti, Gazmend Elezi, Lionel Fadin, Maja Gori, Tobias Krapf, Yannis Maniatis et Charlotte Odie

\section{(2) OpenEdition \\ Journals}

Édition électronique

URL : http://journals.openedition.org/baefe/1362

DOI : $10.4000 /$ baefe.1362

ISSN : 2732-687X

Éditeur

ResEFE

\section{Référence électronique}

Petrika Lera, Gilles Touchais, Cécile Oberweiler, Ole Christian Aslaksen, Charlotte Blein, Athina Boleti, Gazmend Elezi, Lionel Fadin, Maja Gori, Tobias Krapf, Yannis Maniatis et Charlotte Odie, «Bassin de Korçë, Kallamas » [notice archéologique], Bulletin archéologique des Écoles françaises à l'étranger [En ligne], Balkans, mis en ligne le 26 novembre 2020, consulté le 11 décembre 2020. URL : http:// journals.openedition.org/baefe/1362 ; DOI : https://doi.org/10.4000/baefe.1362

Ce document a été généré automatiquement le 11 décembre 2020.

\section{cc) $(1) \&$}

Le Bulletin archéologique des Écoles françaises à l'étranger est mise à disposition selon les termes de la Licence Creative Commons Attribution - Pas d'Utilisation Commerciale - Pas de Modification 4.0 International. 


\title{
Bassin de Korçë, Kallamas
}

\author{
Petrika Lera, Gilles Touchais, Cécile Oberweiler, Ole Christian Aslaksen, \\ Charlotte Blein, Athina Boleti, Gazmend Elezi, Lionel Fadin, Maja Gori, \\ Tobias Krapf, Yannis Maniatis et Charlotte Odie
}

\section{NOTE DE L'AUTEUR}

Composition de l'équipe de terrain : Gilles Touchais (conseiller scientifique), Magali Benet (master, Université de Nantes), Charlotte Blein, Gazmend Elezi, Lionel Fadin, Maja Gori, Ergys Hasa (doctorant, Université de Tirana, responsable administratif de la réserve), Tobias Krapf, Charlotte Odie, Marie Peillet (restauratrice, diplômée de l'INP) et Eduard Shehi (Dr, Institut archéologique de Tirana).

Partenariats institutionnels : Centre d'études albanologiques (Tirana), UMR 7041 « Archéologies et Sciences de l'Antiquité (ArScAn) », Ministère de l'Europe et des Affaires étrangères

Établissements porteurs de l'opération : EFA, Ministère de l'Europe et des Affaires étrangères

Données scientifiques produites : Site de la Mission archéologique francoalbanaise du bassin de Korçë, BCH 119-2 (1995), Chapitre XVII. Sovjan et le lac Maliq en Albanie : Un site protohistorique dans son environnement, Bassin de Korçë, Kallamas

1 La Mission archéologique franco-albanaise du bassin de Korçë, cofinancée par le ministère des Affaires étrangères et l'École française d'Athènes, a poursuivi en 2016 l'étude du matériel et des données des fouilles de Sovjan et de Kallamas, ainsi que des prospections menées dans le bassin de Korçë (programme PALM), en vue de leur publication. Cette nouvelle campagne d'étude, dirigée conjointement par Cécile Oberweiler et Petrika Lera, a réuni en tout une douzaine de personnes, qui ont effectué des séjours plus ou moins longs à Korçë entre le 4 juillet et le 7 août. Les travaux se sont déroulés dans les locaux mis à la disposition de la mission par l'Université de Korçë il y 
a deux ans ${ }^{1}$ mais qui, de façon inattendue, sont passés cette année sous la tutelle de la municipalité. Si ce changement ne remet pas en cause le maintien de la réserve dans le sous-sol du bâtiment ni - pour l'instant du moins - des bureaux occupés par la mission, on peut toutefois craindre qu'il n'affecte le partenariat tout juste noué entre celle-ci et l'université.

\section{L'étude des données des fouilles de sovjan}

2 Comme les années précédentes, on a poursuivi la rédaction du manuscrit du premier volume de la publication, dont deux chapitres sur trois sont maintenant achevés, et les études de matériel qui fourniront la matière des volumes suivants.

\section{La céramique néolithique}

3 En marge de l'étude de la céramique néolithique de Kallamas (v. infra), G. Elezi a examiné de près le petit ensemble de tessons issus des niveaux du Néolithique Moyen de Sovjan (couche 11), plus précisément des sondages A12 (3 tessons) et B2 (30 tessons), soit un total de 33 tessons déjà complètement documentés. Les principales observations qui en ressortent sont les suivantes. La céramique est généralement de couleur sombre (noir, brun, gris). La plupart des tessons noirs sont lissés avec beaucoup de soin, y compris - pour plusieurs fragments appartenant à des formes ouvertes - sur leur face intérieure. Les tessons bruns non décorés sont plus grossièrement lissés, seulement sur leur face extérieure. La face intérieure des tessons appartenant à des formes fermées est simplement égalisée mais, sur certains d'entre eux, elle est complètement érodée. La majorité des fragments appartient à des formes ouvertes (fig. 1) mais on compte aussi quelques formes fermées. L'aspect très fragmenté du matériel ne permet pas de proposer une typologie précise de ces formes. On a toutefois pu identifier un vase sphérique et un autre de forme hémisphérique, l'un des vases fermés étant très probablement à un pot à col. Deux types de décor sont attestés : un décor à la barbotine (6 tessons) et un décor à impresso (4 tessons). Le décor à la barbotine, plus ou moins épais, se présente sous la forme de bandes parallèles, généralement horizontales, situées de préférence sur la partie inférieure du vase. Le décor à impresso est constitué d'empreintes d'ongles ou de doigts (fig. 2) formant des motifs distincts.

Fig. 1. Sovjan, couche 11. Vase du NM de forme ouverte (SV 02/317.2).

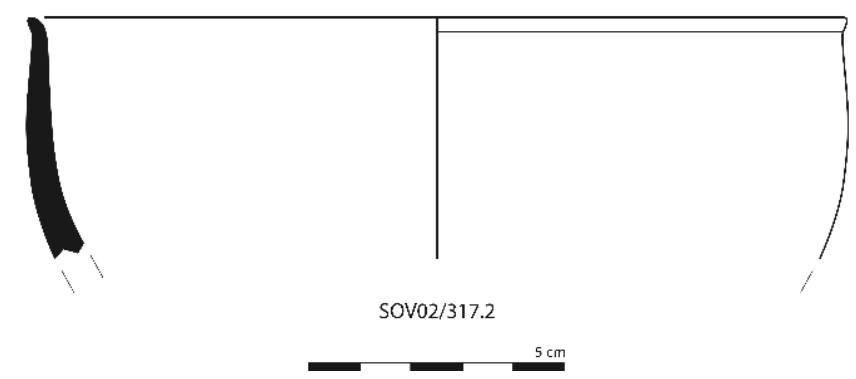

Dessin G. Elezi, DAO M. Benet. 
Fig. 2. Sovjan, couche 11. Tessons NM avec décor à impresso exécuté à l'ongle (a) et au doigt (b).
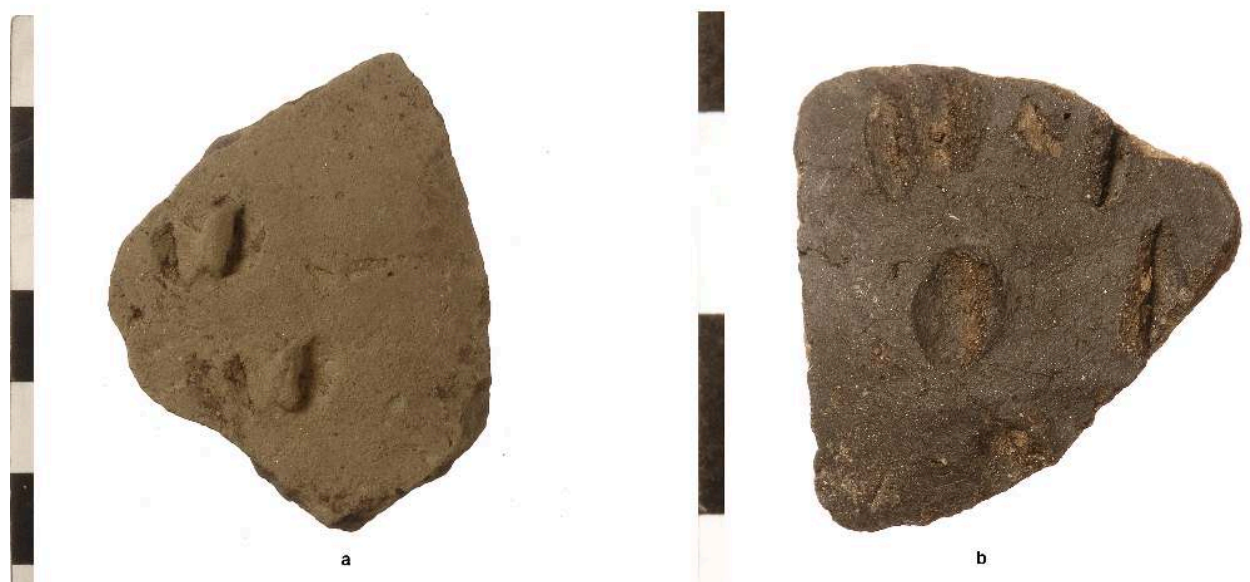

Mission archéologique franco-albanaise du bassin de Korçë.

4 Du point de vue de la technologie, ces vases sont façonnés dans une argile plutôt fine contenant quelques inclusions minérales de petite taille. Le dégraissant utilisé consiste essentiellement en sable, quartz et mica. La couleur noire - totale ou partielle - des tessons indique une cuisson en atmosphère réductrice.

Quoique peu abondant, ce matériel présente les traits morpho-technologiques typiques de la céramique du NM connue dans la région (Dunavec, Luadishtë). Elle appartient cependant à une phase plus ancienne que la céramique du niveau NM de Kallamas², comme l'indiquent à la fois les datations absolues (celles de la couche 11 de Sovjan sont antérieures de près d'un millénaire à celles du premier niveau de Kallamas, v. infra) et la typologie : le profil hémisphérique du vase de la fig. 1 s'inscrit clairement dans la tradition de la phase « développée » du NA albanais ${ }^{3}$.

\section{La céramique de l'Âge du Bronze et du Fer}

6 Tout en poursuivant leurs recherches respectives sur la céramique des niveaux des âges des métaux de Sovjan, M. Gori et T. Krapf ont uni leurs efforts pour élaborer une première synthèse sur la séquence céramique de ces périodes publiée dans la revue Iliria ${ }^{4}$.

\section{La céramique du Bronze Ancien final et du Bronze Moyen}

7 Dans le cadre de l'étude technologique de la céramique des couches 7, 8 et 9, M. Gori a procédé cette année au contrôle et au prélèvement de nouveaux échantillons de céramique représentatifs de ces trois couches, sur la base des résultats des analyses par spectromètre XRF portatif réalisées l'an dernier par 0 . Aslaksen ${ }^{5}$. L'objectif est de compléter ces premiers résultats par une série d'analyses pétrographiques et chimiques, qui devraient être réalisées au Fitch Laboratory de l'École britannique d'Athènes. M. Gori a d'autre part profité de son séjour pour examiner la céramique des mêmes périodes recueillie au cours des sept campagnes de prospection du programme PALM (v. infra). 


\section{La céramique du Bronze Récent et du Fer Ancien}

De son côté, T. Krapf a poursuivi, dans le cadre de sa thèse, l'étude de la céramique des couches 4,5 et 6 , effectuant une nouvelle série de photos au microscope des différentes pâtes céramiques et complétant le dossier graphique du matériel. D'autre part, grâce au travail de restauration effectué sur les vases du sondage B3 (fig. 3), il apparaît désormais clairement que la céramique des niveaux inférieurs de ce sondage doit être assignée à la couche 6 , comme cela avait déjà été soupçonné 6 . T. Krapf a rendu compte des derniers résultats de ses travaux dans deux communications ${ }^{7}$. Le succès du premier séminaire itinérant qu'il avait organisé en $2015^{8}$ l'a en outre poussé à en organiser un second cette année, en collaboration avec 0 . Aslaksen?.

Fig. 3. Sovjan. Tesson à peinture mate : section et détail de la peinture en surface.
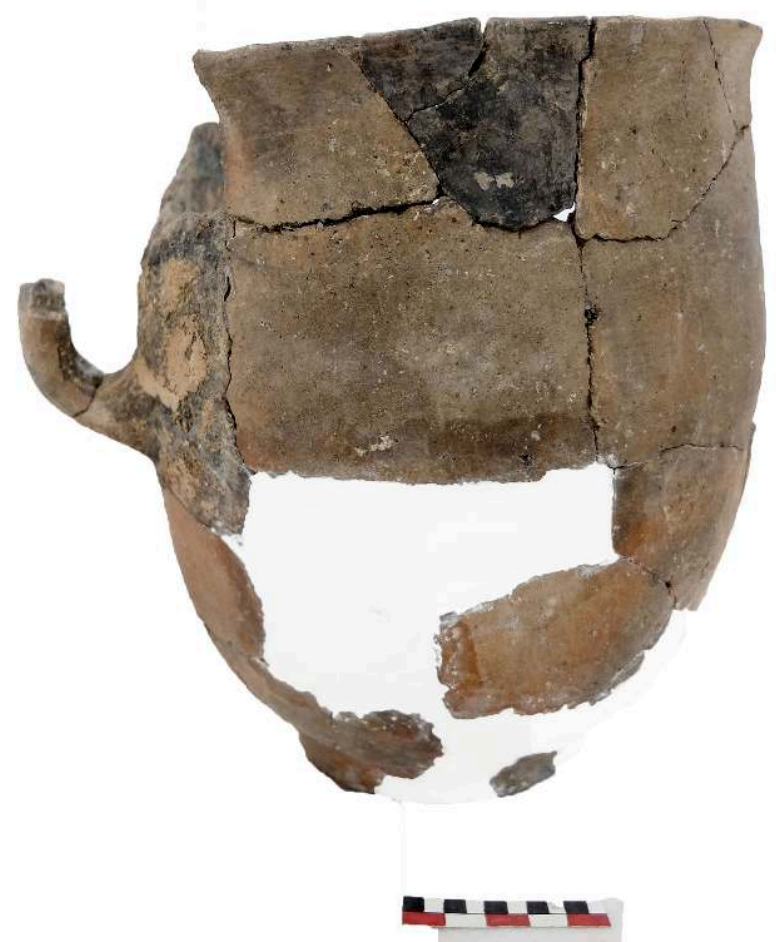

Clichés T. Krapf.

\section{Les analyses par spectrométrie XRF}

O. Aslaksen a complété, avec la collaboration de T. Krapf et M. Gori, les premières analyses par spectrométrie XRF qu'il avait réalisées pendant l'été 2015 sur la céramique de l'Âge du Bronze et du Fer de Sovjan ${ }^{10}$. Une série de 451 mesures supplémentaires a pu être recueillie et incluse dans la base de données élaborée à partir des premières mesures de Sovjan et de mesures faites sur du matériel provenant de plusieurs sites d'Épire (Grèce). L'inclusion de la céramique du BA de Sovjan a permis d'élargir le spectre chronologique couvert jusqu'ici par les analyses et d'observer des différences de composition entre les échantillons du BA et du BR. En général, les échantillons des phases plus anciennes contiennent moins de $\mathrm{Fe}$ et de $\mathrm{Cr}$ que ceux du BR, mais il y a toutefois de nombreuses similitudes de composition entre les deux périodes. D’autre 
part, la céramique du BR présente une plus grande variabilité, ce qui s'accorde avec les résultats de l'étude macroscopique. La base de données des compositions céramiques rassemble aujourd'hui un total de 1400 mesures, dont 995 provenant du matériel de Sovjan et 405 de celui de sites épirotes. Cette base de données a désormais une portée régionale puisqu'elle couvre le Nord-Ouest de la Grèce et le Sud-Est de l'Albanie. On prévoit d'y intégrer pour comparaison des mesures faites sur des échantillons d'argile.

10 À partir des résultats préliminaires, on peut formuler plusieurs observations. Tout d'abord, les mesures faites sur les tessons mycéniens montrent une teneur élevée en chrome $(\mathrm{Cr})$ et en fer $(\mathrm{Fe})$ dans les zones peintes, par comparaison avec les zones non décorées. Il en est de même pour une grande partie des tessons de céramique à peinture mate (fig. 4), même si certains tessons présentent dans les zones peintes un taux de $\mathrm{Cr}$ plus bas, proche de celui des zones sans peinture. Cette première observation doit cependant être validée par un examen plus approfondi. D'autre part, tandis que certains éléments comme $\mathrm{K}, \mathrm{Fe}, \mathrm{Ca}, \mathrm{Mn}, \mathrm{Cr}, \mathrm{Ti}, \mathrm{Sr}, \mathrm{Rb}$ et $\mathrm{Zr}$ apparaissent dans les 995 mesures réalisées (fig. 5), d'autres éléments, comme Ag et Mo, sont beaucoup plus rares. Des recoupements dans la composition de différents groupes de vases décorés peuvent aussi être observés dans certains cas, mais cela doit être confirmé par un travail analytique plus poussé, actuellement en cours. Ces résultats préliminaires ont fait l'objet d'un poster présenté au $41^{\mathrm{e}}$ Colloque international d'archéométrie de Kalamata $^{11}$.

Fig. 4. Sovjan, couche 6 . Vase de stockage en cours de restauration.

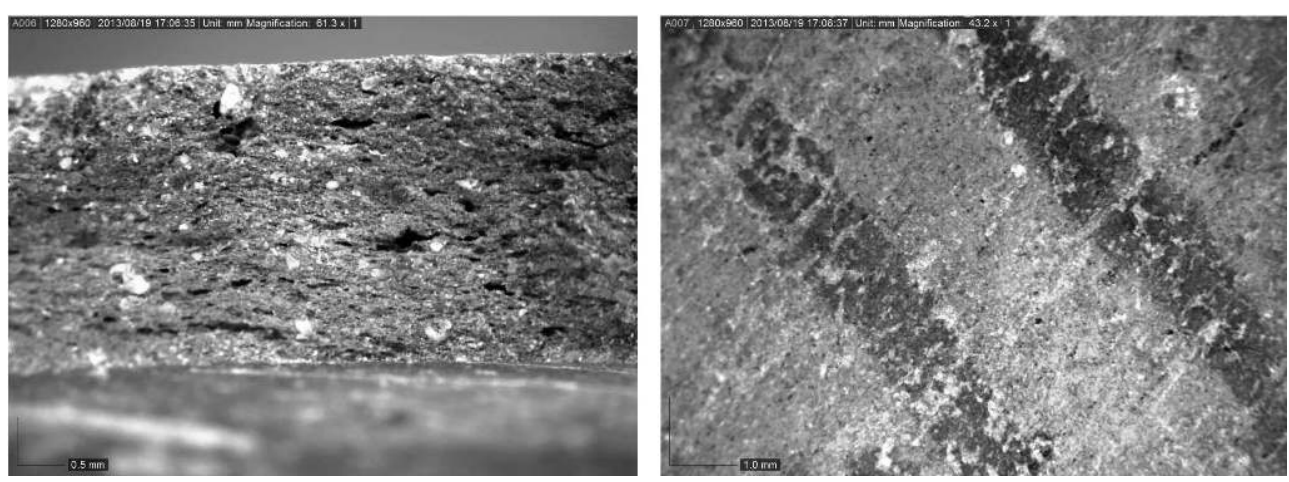

Mission archéologique franco-albanaise du bassin de Korçë. 
Fig. 5. Analyses XRF : fréquence comparée des éléments présents dans les pâtes céramiques de Sovjan.

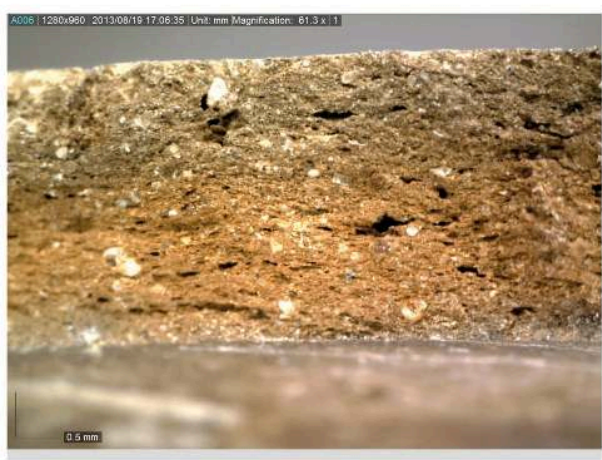

Matt painted sherd: section and detail (Photos: Tobias Krapf)

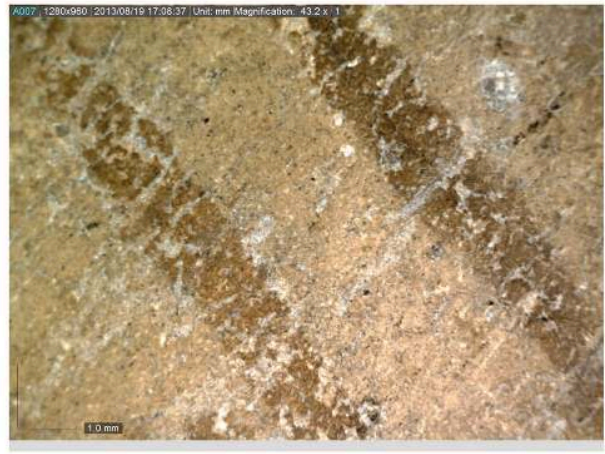

Graphique O. Aslaksen.

\section{L'étude des données des fouilles de Kallamas}

L'effort principal a porté cette année sur l'étude de la céramique, qui a été entreprise par G. Elezi avec l'aide de P. Lera, tandis que A. Boleti, qui n'a pu se rendre à Korçë cette année, poursuivait à distance celle des lames en pierre polie. D'autre part, la chronologie absolue des phases d'occupation du site a fait l'objet d'une modélisation.

\section{La chronologie absolue}

Y. Maniatis a repris les huit datations absolues obtenues jusqu'à présent ${ }^{12}$ afin de proposer un modèle chronologique plus précis pour l'ensemble des niveaux d'occupation du sondage principal $\mathrm{C}$, où la séquence d'occupation est la plus longue (fig. 6). On obtient ainsi un découpage chronologique moyen pour chaque phase :

\begin{tabular}{|c|c|}
\hline Phase & Date moyenne \\
\hline Début de l'occupation & $\sim 5203 \mathrm{BC}$ \\
\hline Transition III/II & $\sim 5142 \mathrm{BC}$ \\
\hline Transition II/IC & $\sim 5097 \mathrm{BC}$ \\
\hline Transition Ic/Ib & $\sim 4981 \mathrm{BC}$ \\
\hline Fin de l'occupation & $\sim 4562 \mathrm{BC}$ \\
\hline
\end{tabular}


Fig. 6. Séquence des datations absolues de Kallamas après modélisation.

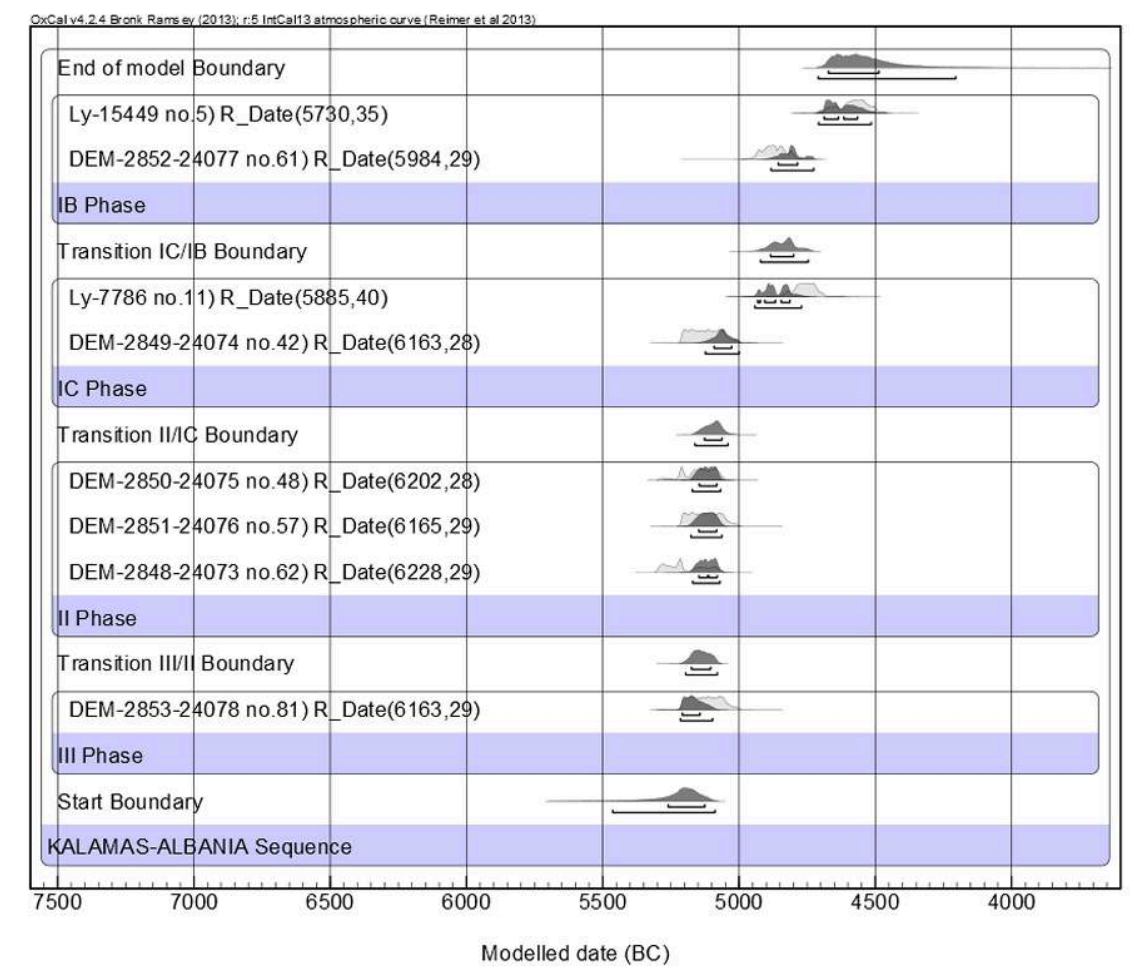

Mission archéologique franco-albanaise du bassin de Korçë.

13 On constate donc, après lissage, que ces niveaux correspondent bien à des phases d'occupation humaine très rapprochées, comme on l'avait déjà soupçonné. La durée moyenne de la phase III est ainsi de 61 ans, celle de la phase II de 45 ans et celle de la phase Ic de 116 ans. Seule la phase Ib est plus longue, avec une durée moyenne de 419 ans, mais cela est probablement dû à la nature de l'échantillon analysé puisqu'il s'agit de deux échantillons à durée de vie longue (charbons de bois), tandis que les autres sont à durée de vie courte (graines). Les autres datations absolues obtenues dans le sondage D1, supposées un peu plus anciennes que celle du niveau III de C1, sont en réalité à peu près contemporaines. Cela indiquerait, dans l'état actuel de l'exploration du site, que la première implantation humaine sur le site de Kallamas daterait des environs de $5200 \mathrm{BC}$, c'est-à-dire du Néolithique Récent I en termes de chronologie égéenne.

\section{La céramique}

G. Elezi a procédé à l'enregistrement du matériel de 61 unités stratigraphiques (US) appartenant à cinq sondages différents : C1 (16 US), C8 (13 US), C9 (9 US), D1 (16 US) et D11 (7 US). Parallèlement à ce travail, il a procédé, avec l'aide de la restauratrice, à la recherche de recollages entre tessons d'une même US ou d'US différentes afin de reconstituer le plus grand nombre possible de profils ou de formes complètes.

Sur l'ensemble du matériel examiné, 82 tessons ont été dessinés (fig. 7) et 15 échantillons sélectionnés aux fins d'analyses pétrographiques; ces analyses, qui visent à éclairer les questions relatives à la production, à la technologie et à la circulation de la céramique de Kallamas, doivent être réalisées au département de 
géologie de l'université de Thessalonique. Treize autres tessons qui présentent des résidus organiques adhérant à leur face intérieure (fig. 8) ont été prélevés aux fins d'analyses chimiques, afin de déterminer la fonction des vases auxquels ils appartiennent.

Fig. 7. Kallamas. Vases KAL 11/153.27.
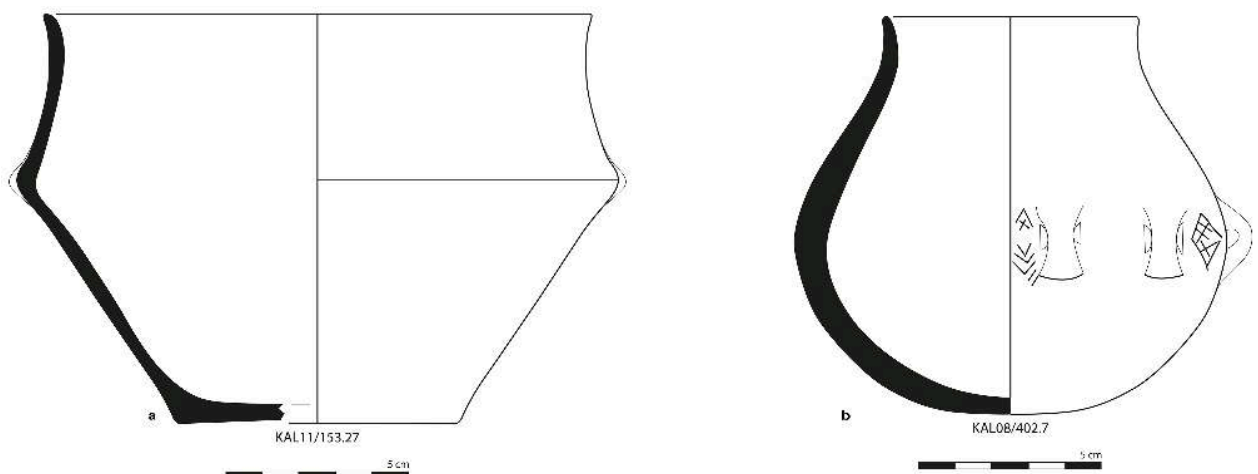

Dessin G. Elezi, DAO M. Benet.

Fig. 8. Kallamas. Tesson avec résidus organiques sur sa face intérieure (KL 11/346.1).

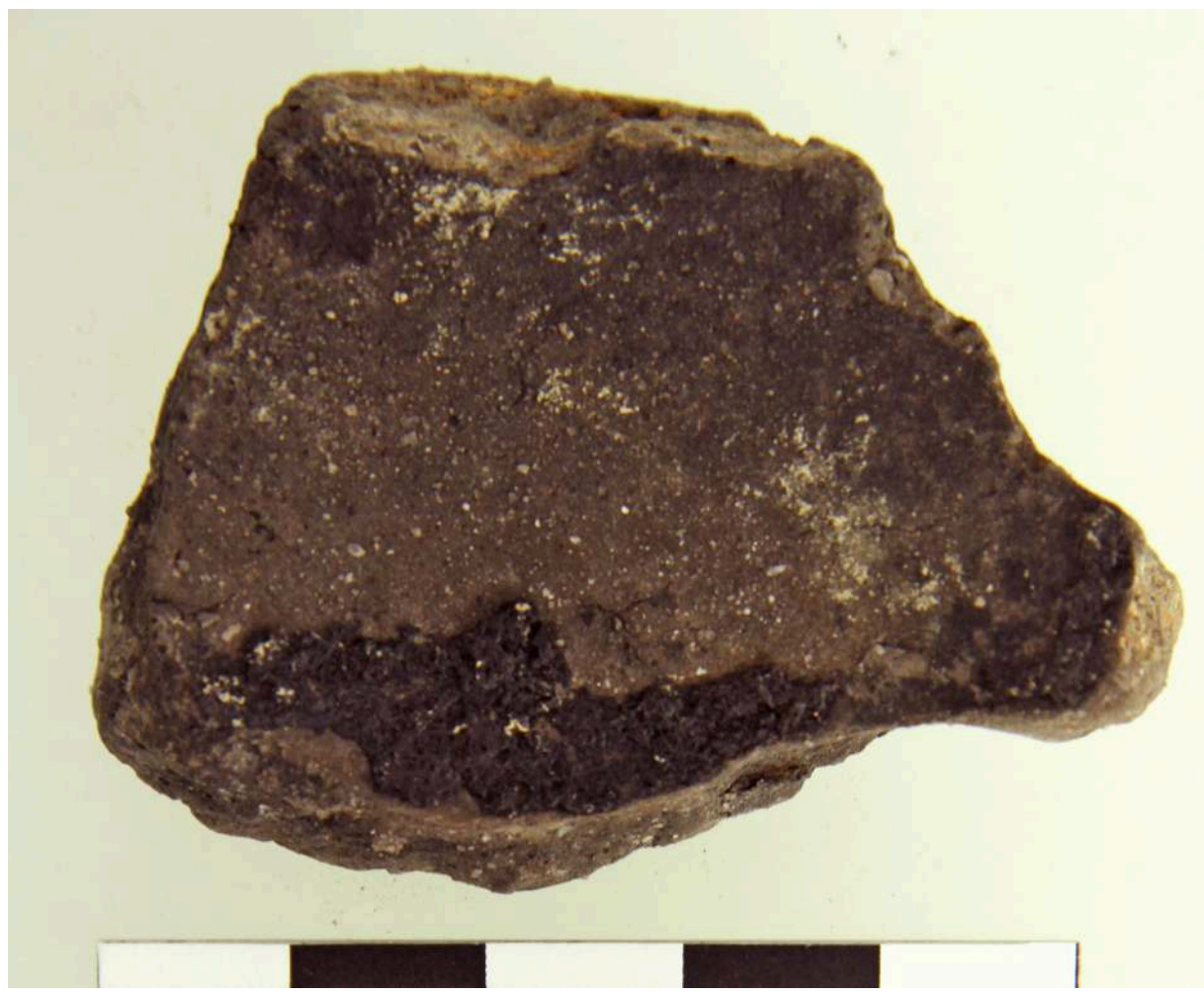

Mission archéologique franco-albanaise du bassin de Korçë.

\section{L'outillage en pierre polie}

A. Boleti a poursuivi l'étude technologique des lames polies de Kallamas, qui vise in fine à reconstituer les chaînes opératoires de production et de consommation de ces outils, et aussi à retracer les réseaux d'échanges dont ils font partie. Bien que le bilan définitif 
ne puisse pas être encore dressé, quelques apports de l'étude en cours méritent d'être soulignés.

Deux grands groupes des matières premières utilisées pour la production de ce type d'outillage avaient été distingués au début de l'étude : 1) des roches de la famille des «syénites"; 2) d'autres types de roches, définis avec moins de précision. On sait désormais que la grande majorité des roches du deuxième groupe appartient à la famille des néphrites (fig. 9). L'identification a été faite sur des critères macroscopiques, après comparaison avec des pièces en néphrite fournies par P. Pétrequin (Projet JADE) et provenant d'autres sites. Bien que les gisements de néphrite restent à localiser, Kallamas s'inscrit dans un réseau important de diffusion de ce matériau.

Fig. 9. Kallamas. Exemples de haches polies en néphrite.

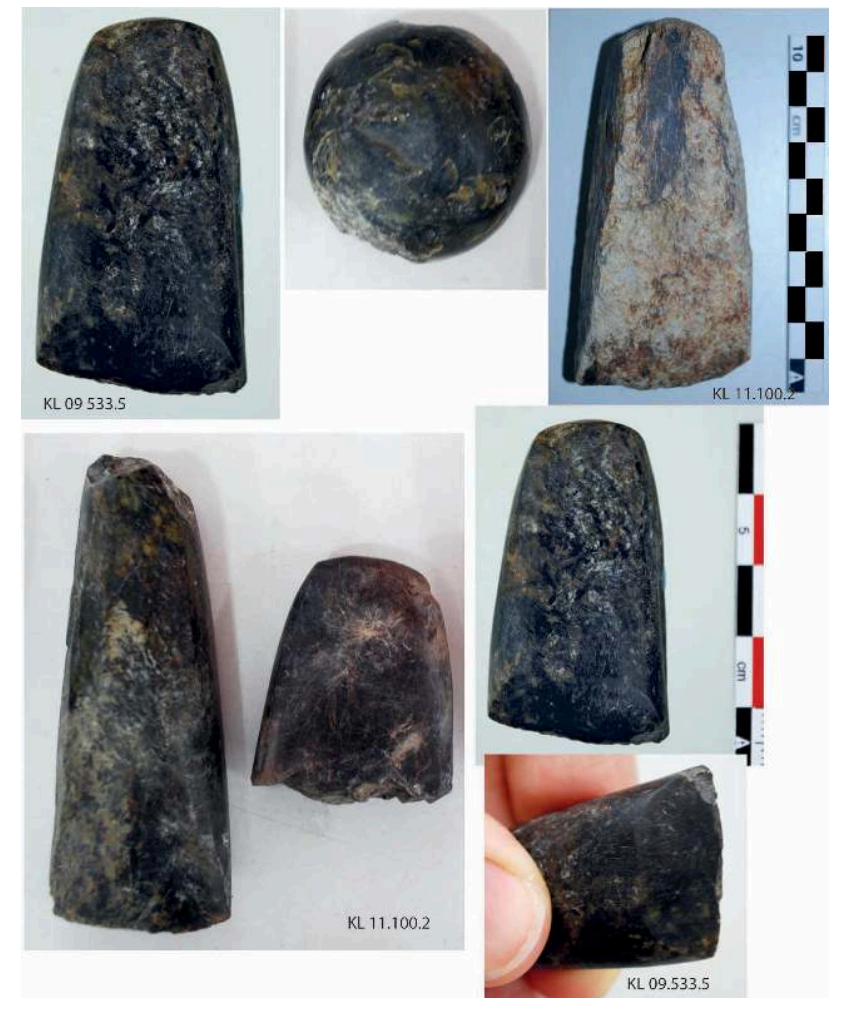

Mission archéologique franco-albanaise du bassin de Korçë.

L'étude des techniques de production des lames polies en "syénites », notamment des techniques de polissage, fait actuellement l'objet d'une étude plus approfondie. Des analyses des surfaces des lames de cette série sont prévues au Laboratoire de Tribologie et de Dynamique des Systèmes (LTDS) de l'École Centrale de Lyon. Ces analyses (notamment la caractérisation multi-échelle par la méthode des ondelettes continues) permettront d'évaluer l'état de la surface (poli de haute qualité d'après l'observation macroscopique) à différentes échelles et contribueront à la reconstitution des techniques, après comparaison avec les surfaces polies expérimentales ${ }^{13}$, mais aussi avec les surfaces de lames polies ethnographiques. 


\section{L'étude des données du programme PALM}

19 L'élaboration des résultats des prospections menées, entre 2007 et 2013, dans la partie Nord du bassin de Korçë, s'est poursuivie cette année. Toutefois, les contraintes budgétaires n'ont pas permis d'entreprendre l'opération de terrain qui avait été envisagée (prospection systématique avec ramassage du matériel sur certains sites en prévision de fouilles futures). On s'est donc concentré sur trois tâches : d'une part, les études de matériel, les travaux de documentation et l'élaboration des données ; d'autre part, l'inventaire et le géo-référencement des sites antiques déjà connus hors de la zone prospectée par le programme PALM, dans le cadre d'une réflexion historique plus globale amorcée par Ch. Blein et E. Shehi depuis 2013 ; enfin, la refonte du SIG PALM pour le rendre utilisable en Web SIG.

\section{La céramique de l'Âge du Bronze}

M. Gori s'est engagée, en collaboration avec T.Krapf, dans l'étude du matériel céramique de l'Âge du Bronze issu du programme PALM. Elle s'est plus particulièrement consacrée, cette année, à l'étude de la céramique du site 17-81.1, découvert en 2009 au sud du village de Vreshtasi et sur lequel deux petits sondages stratigraphiques de contrôle avaient été réalisés en $2010^{14}$, livrant une céramique suffisamment abondante pour permettre une étude typo-chronologique substantielle. Une partie de ce matériel avait déjà été partiellement documentée à l'issue des fouilles. Cette année le travail de documentation a été achevé, l'ensemble de la céramique étant photographié et dessiné (fig. 10) et les précédents dessins contrôlés.

Fig. 10. PALM, site 17-81.1.

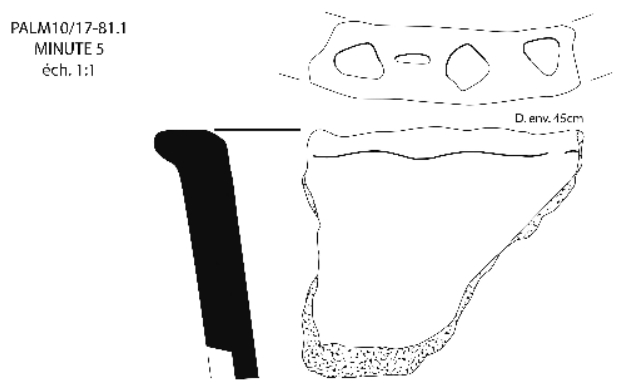

V12 - PALM10/111.11

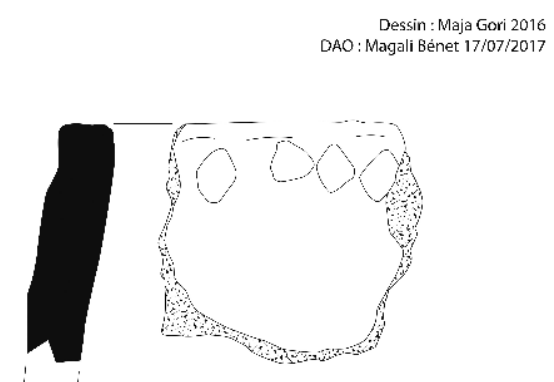

V13- PALM10/111.17

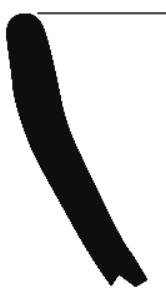

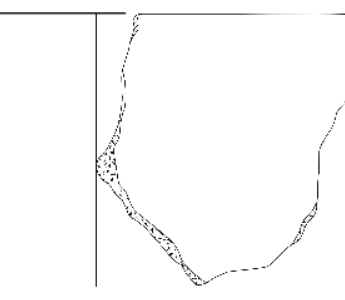

V14 - PALM10/111.23
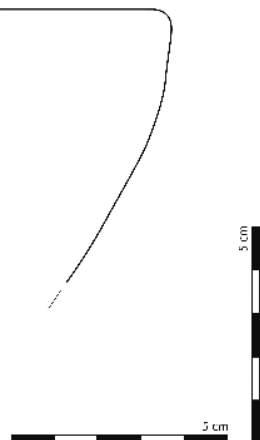

Mission archéologique franco-albanaise du bassin de Korçë. 
Cet examen a permis de faire un certain nombre d'observations préliminaires. D'une manière générale, la céramique est assez mal conservée, à l'exception de quelques tessons provenant de l'US 112. Elle est entièrement façonnée à la main, dans une pâte plutôt grossière contenant souvent de grosses inclusions minérales. La coloration superficielle des vases, simultanément grise et rouge, indique une absence ou un défaut de contrôle de l'atmosphère de cuisson, à la fois réductrice et oxydante. La surface des vases est généralement rugueuse, mais quelques fragments présentent des traces de lissage.

L'ensemble du matériel semble appartenir à un contexte chronologique homogène, sous réserve d'une analyse typologique approfondie, qui permettra d'en préciser les caractéristiques chrono-culturelles. Par comparaison avec la céramique des couches 7 et 8 de Sovjan, celle de Vreshtasi est incontestablement plus ancienne. Cela s'accorde avec les deux dates ${ }^{14} \mathrm{C}$ obtenues en 2012, qui placent l'occupation du site au tout début du $3^{\text {e }}$ millénaire av. J.-C. soit au début du Bronze Ancien ${ }^{15}$.

\section{La céramique antique}

E. Shehi a rédigé un premier jet du texte sur la céramique antique pour la publication finale, avec références aux planches de dessins déjà réalisées. Il reste à terminer le catalogue, en essayant de préciser la chronologie de chaque tesson, et à apporter les derniers compléments aux planches. D'autre part, le travail de documentation sur la céramique issue des anciennes fouilles albanaises dans le bassin de Korçë a été poursuivi (dessins, photos, prélèvements d'échantillons), mais la plupart des tessons conservés sont malheureusement dépourvus d'indications de provenance.

\section{L'Inventaire des sites antiques du bassin de Korçë}

Ch. Blein a poursuivi, en collaboration avec P. Lera, l'inventaire des sites antiques du bassin de Korçë entrepris l'an dernier, menant de front reconnaissance pédestre et implémentation de la base documentaire. D'autre part, une courte mission topographique a permis à L. Fadin de procéder au relevé par drône de deux des sites reconnus l'an dernier.

\section{Reconnaissances pédestres}

L'équipe, composée de Ch. Blein, P. Lera, M. Benet et E. Rakipllari, a achevé la couverture de la partie Sud du bassin, commencée l'an dernier, et entamé celle de la partie Nord.

Dans la partie Sud, les sites fortifiés de Dishnica et Hija e Korbit ont été visités, ainsi que Malaveci, qui est probablement un habitat d'époque romaine (fig. 11). On a par ailleurs tenté de contrôler la présence de matériel archéologique dans une petite zone urbaine qui s'étend du quartier Sud-Est de Korçë à Mborja, zone dans laquelle divers vestiges avaient été découverts il y a une trentaine d'années, suggérant l'existence d'un établissement antique. Mais les nombreuses constructions qui s'y sont développées depuis, ainsi que les parcelles clôturées, ont rendu cette entreprise infructueuse. 
Fig. 11. Sites antiques inventoriés et géo-référencés en 2015 et 2016.

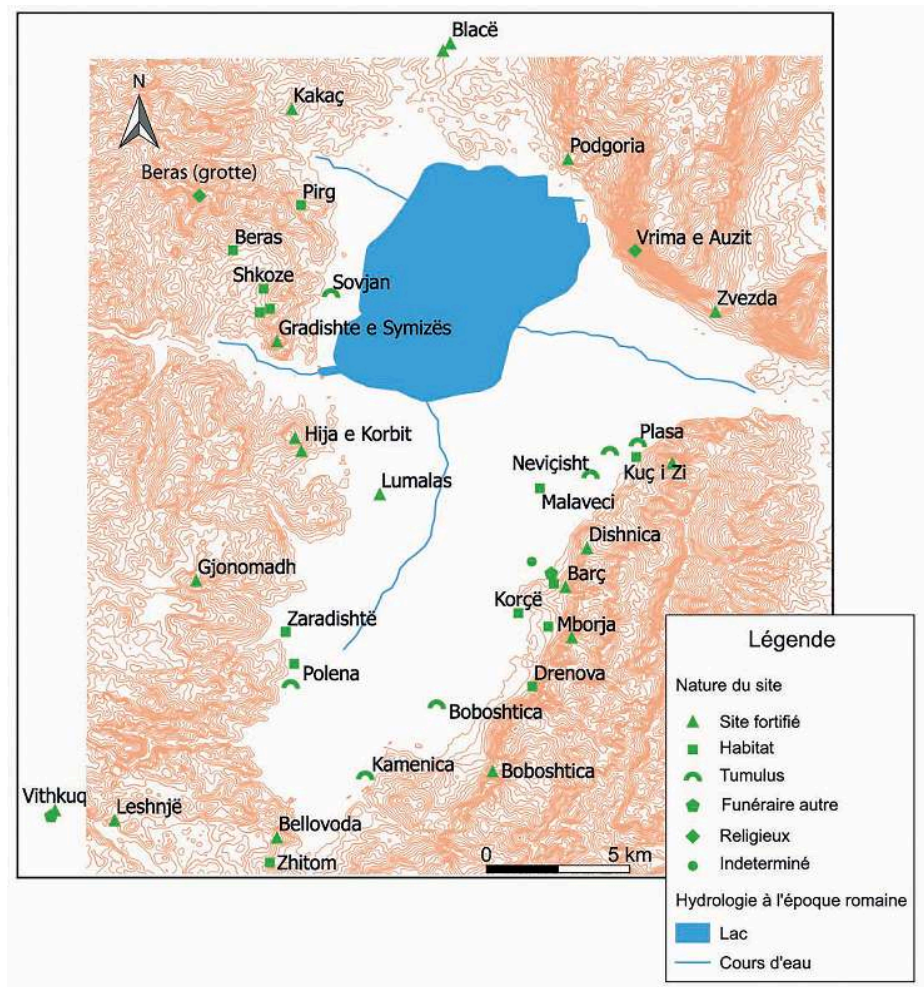

Carte extraite du SIG PALM

27 À Hija e Korbit, on a relevé des traces de fouilles clandestines récentes : un trou de plusieurs mètres de profondeur avait été creusé dans le mur de fortification tandis que, sur le plateau, la terre avait été retournée jusqu'à $50 \mathrm{~cm}$ de profondeur environ sur plusieurs centaines de mètres carrés. Le site porte également les traces de fouilles clandestines plus anciennes. Ces actes sont particulièrement regrettables s'agissant d'un site de cette importance, que l'on a proposé d'identifier avec l'antique Pélion et où l'on a enregistré des découvertes fortuites notables ${ }^{16}$.

Dans la partie Nord du bassin, on a visité les sites de Berasi, Blacë, Gradishta (Symize), Kakaç, Kembë Thekri, Pirgu, Shkoze et Zvezda (fig. 11). Là encore, on a pu observer des traces de fouilles clandestines en plusieurs endroits, moins systématiques toutefois qu'à Hija et Korbit. Comme l'an dernier, tous les sites visités cette année ont été géoréférencés afin d'apparaître dans le Web SIG de PALM (v. infra).

Ce travail d'inventaire amène a formuler plusieurs remarques, malgré les obstacles, déjà soulignés l'an dernier, auxquels se heurte l'interprétation : chronologie imprécise des sites et relevés trop peu nombreux.

Tout d'abord, l'exploration de la partie Nord du bassin permet d'affirmer que l'ensemble des pourtours de ce dernier était jalonné d'établissements fortifiés, juchés au sommet de collines plus ou moins élevées et escarpées. Comme on l'a noté l'an dernier, les établissements fortifiés de la partie Sud-Est du bassin, sur les flancs du mont Morava, sont implantés à intervalles à peu près réguliers, permettant presque un relais visuel continu ; le site de Dishnica vient compléter le tableau (fig. 12). 
Fig. 12. Panorama du massif de la Morava avec les sites fortifiés.

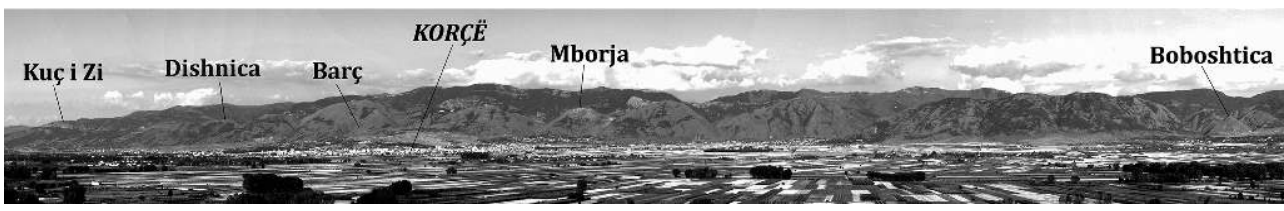

Cliché Ch. Blein. Berasi et de Shkoze, au Nord de Maliq et à l'Est de Sovjan, montrent clairement que les piémonts des massifs montagneux délimitant le bassin, constitués de collines propices à l'agriculture étaient également habités (fig. 11).

notera la présence de deux grottes présentant des traces d'occupation dans la partie Nord de la plaine : l'une sur la commune de Berasi, l'autre - dénommée Vrima e Auzit - sur les hauteurs de Gurbardhë ; cette dernière est visiblement un lieu de culte. Vu son emplacement, il est probable que celle de Berasi a eu la même fonction, mais aucune découverte ne vient appuyer cette hypothèse. En tout cas, ce type de site n'est jusqu'à présent pas connu dans la partie Sud du bassin.

\section{Base documentaire}

Afin de rassembler la documentation relative à chaque site et de préserver un maximum d'informations, l'ensemble des archives iconographiques (photos de fouilles et de mobilier) accumulé par P. Lera tout au long de sa carrière a été dépouillé, identifié et légendé. Toute cette documentation relative au bassin de Korçë et à la vallée du Devoll a ainsi pu être classé par site. Les tirages argentiques ont été numérisés par E. Hasa.

\section{Relevés par drone}

Pendant la première semaine de la campagne, L. Fadin a réalisé le relevé photographique par drone de trois sites fortifiés visités l'an dernier : Bellovoda, Mborja et Boboshtica (fig. 11). Ces sites présentaient d'imposants vestiges bâtis (murs de fortification, tours et autres constructions). L'enjeu était d'enregistrer les informations encore visibles aujourd'hui ${ }^{17}$, sachant que toute exploration plus poussée (étude, fouille) serait rendue difficile par l'état et la situation géographique des sites. Il s'agissait par ailleurs d'obtenir des vues aériennes générales des sites, afin de tenter de préciser le plan des vestiges.

Des trois sites dont on avait prévu le relevé, seuls Bellovoda et Mborja ont pu être traités ; il n'a pas été possible d'accéder au site de Boboshtica car le sommet sur lequel il se trouve a été " privatisé » par des habitants du village, ce qui en interdit l'accès.

Pour la réalisation de ces relevés photographiques, on disposait d'un DGPS de marque Leica de type GS15 et d'un drone de marque Hélicéo de type hexacoptère muni d'un appareil photographique Sony A6000. La procédure était la suivante : on commence par positionner des cibles carrées de $50 \mathrm{~cm}$ de côté (fig. 13) aux quatre coins et au centre de la zone à relever, puis le drone survole le site à quatre reprises en prenant une photo tous les $5 \mathrm{~m}$ (fig. 14). Ces photos sont ensuite assemblées pour produire des orthophotos (fig. 15-16) ainsi que des images 3D des sites. Pour le moment, seule la 
cohérence du modèle a été testée et une orthophotographie de basse qualité a été produite (fig. 17). Il reste à calculer la position précise des cibles en post-traitement, géo-référencer les cibles visibles sur les clichés et relancer des calculs avec des paramètres réglés en haute qualité.

Fig. 13. Cible topographique pour le relevé du site de Mborje.

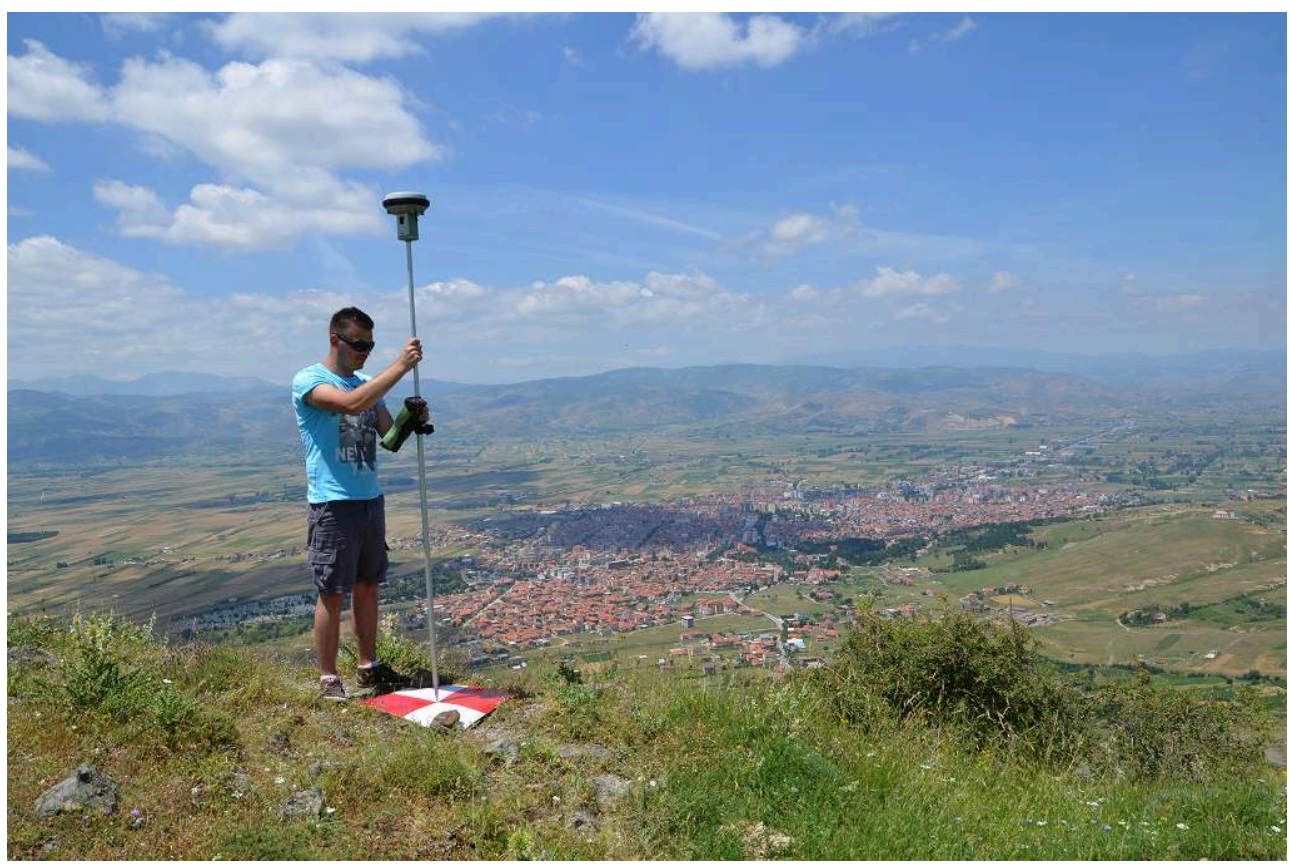

Mission archéologique franco-albanaise du bassin de Korçë.

Fig. 14. Drone en vol procédant au relevé du site de Mborje.

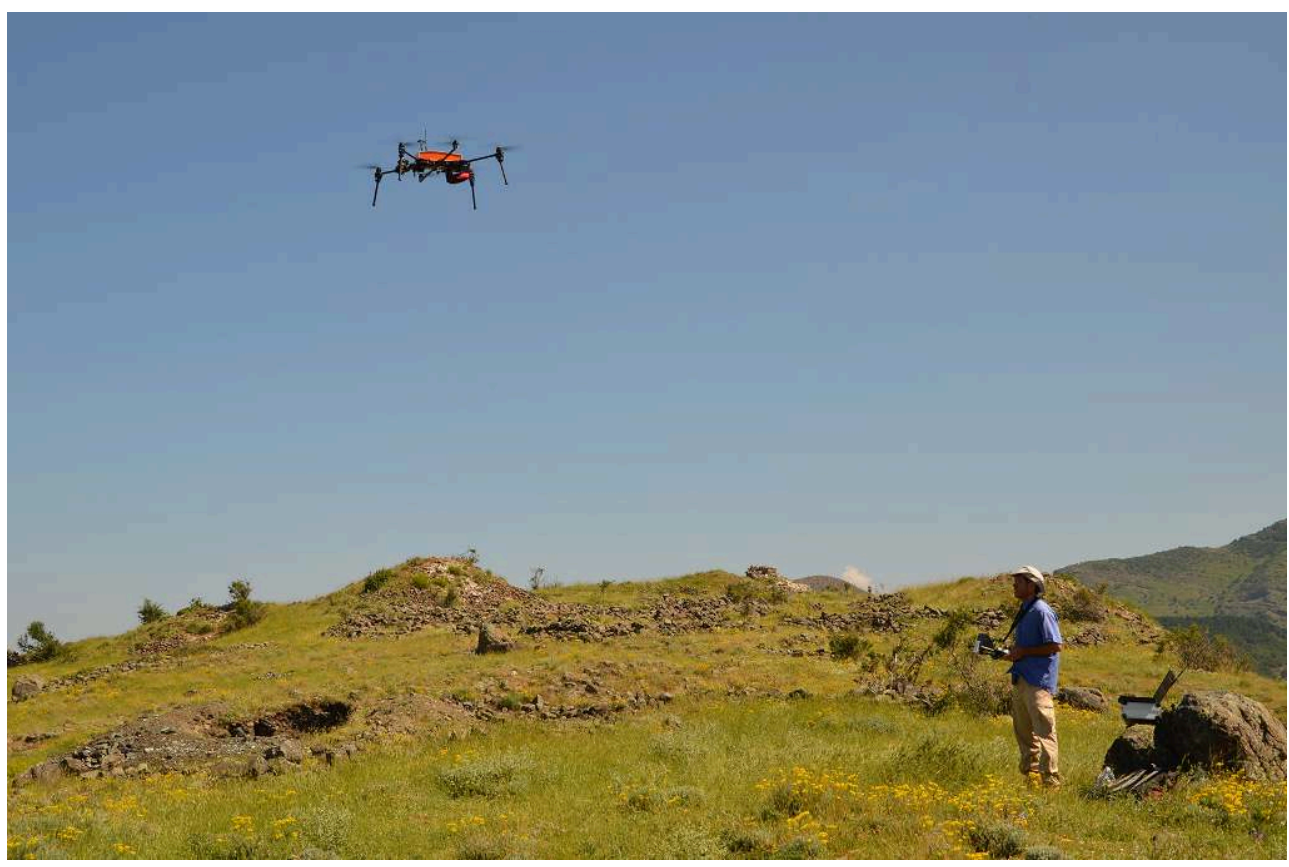

Mission archéologique franco-albanaise du bassin de Korçë. 
Fig. 15. Photo aérienne des vestiges de Mborje.

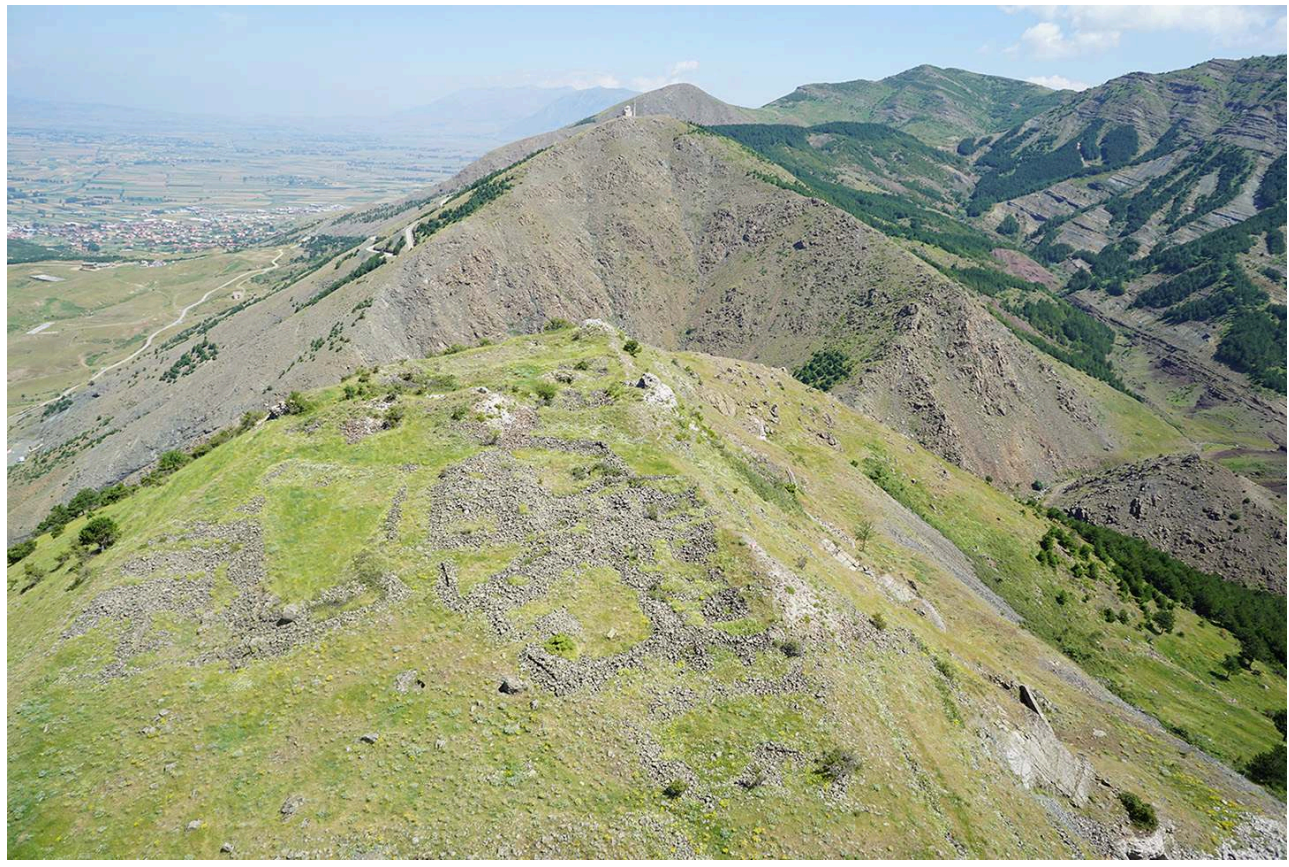

Mission archéologique franco-albanaise du bassin de Korçë.

Fig. 16. Photo aérienne des vestiges de Bellovode.

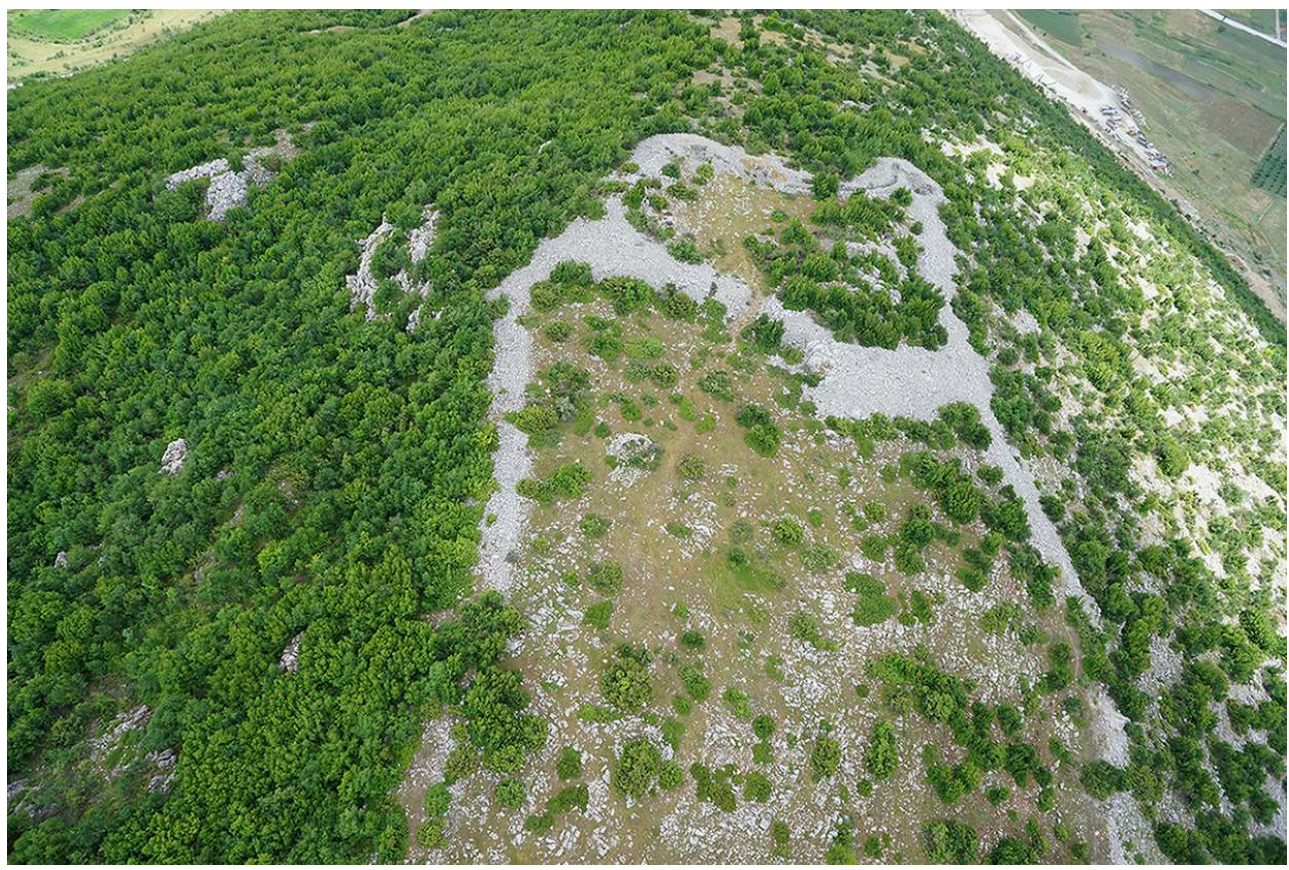

Mission archéologique franco-albanaise du bassin de Korçë. 
Fig. 17. Relevé préliminaire final 3D du site antique fortifié de Mborje à partir des ortophotos.

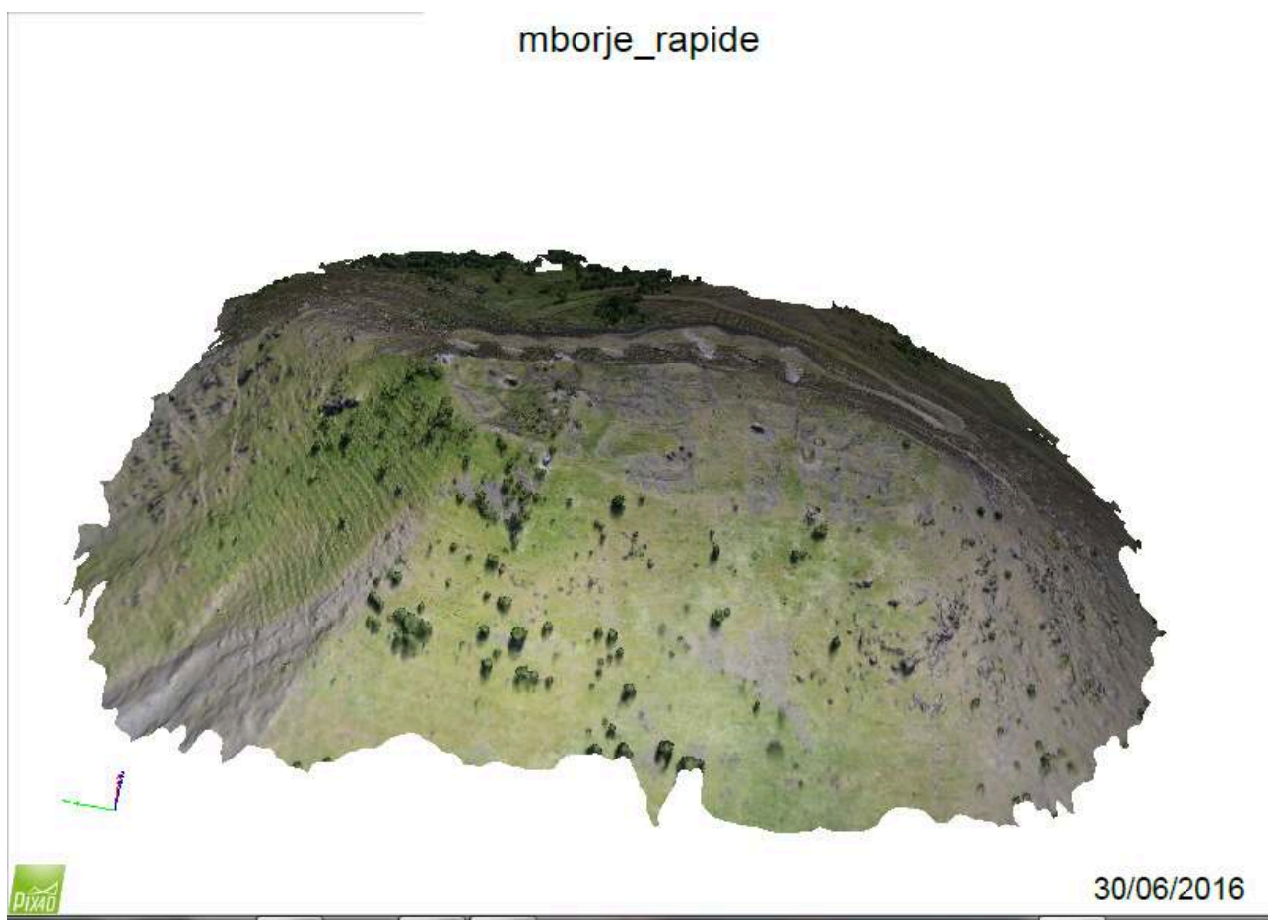

Mission archéologique franco-albanaise du bassin de Korçë.

\section{Perspectives}

Pour mener à bien l'inventaire des sites antiques situés hors de la zone de prospection $\mathrm{du}$ programme PALM et recueillir les informations minimales nécessaires à une meilleure interprétation de ces derniers, deux tâches principales doivent maintenant être accomplies : d'une part, poursuivre le relevé photographique par drone des sites où les vestiges sont apparents (p. ex. Blacë, Hija e Korbit); d'autre part, prospecter systématiquement les sites où l'on a observé la présence de céramique en surface afin d'en établir la chronologie de leur occupation.

\section{Finalisation du SIG PALM en Web SIG}

Ch. Odie a consacré son mois de stage, d'une part, à la ré-harmonisation de l'ensemble des données destinées à apparaitre dans le SIG, d'autre part, à la mise en forme des nouvelles données ${ }^{18}$.

Il s'agissait d'abord de reprendre toutes les données topographiques, géologiques, géomorphologiques et paléo-environnementales pour les vérifier et les harmoniser, afin de recréer un fichier SIG sous QGIS débarrassé des erreurs que l'on y avait détectées et qui constituera le fichier source pour sa transformation en Web SIG. Une fois ce travail achevé, les deux bases de données de PALM (unités de prospection et sites) ont été refondues, puis une nouvelle base de données (sites antiques de l'ensemble du bassin de Korçë) a été créée à partir du travail de Ch. Blein. La charte graphique a également été améliorée. 
40 Nous disposons donc maintenant d'un fichier SIG PALM sous QGIS parfaitement à jour, qu'il reste à convertir en Web SIG. Ce travail doit être réalisé par le service informatique de l'EFA.

\section{Conclusion}

41 Malgré une diminution très sensible des crédits alloués cette année à la mission et les incertitudes qui pèsent sur la pérennité des locaux qu'elle occupe à Korçë, l'équipe de recherche a réussi à maintenir son activité scientifique à un niveau décent - une activité qui est désormais axée en priorité sur l'élaboration, l'interprétation et la diffusion des résultats des travaux de terrain. L'avancement des publications demeure toutefois tributaire du statut précaire de plusieurs membres de l'équipe, qui, malgré tous leurs efforts, ne peuvent pas consacrer à cette tâche tout le temps qui serait nécessaire.

\section{BIBLIOGRAPHIE}

ASLAKSEN, GORI, KRAPF 2016

Ole Christian Aslaksen, Maja Gori, Tobias Krapf, « Characterising Bronze and Early Iron Age ceramics of Sovjan (SE Albania) with the use of pXRF-scanning », $41^{\text {st }}$ International Symposium on Archaeometry (ISI), Kalamata, 15-21 mai 2016.

GJONGECAJ 1985

Shrepsa Gjongecaj, « Le trésor de Hija e Korbit », Iliria 1, 1985, p. 167-209.

GORI 2015

Maja Gori, Along the Rivers and Through the Mountains. A revised chrono-cultural framework for the south-western Balkans during the late 3rd and early 2nd millennium BCE, Bonn, Verlag Rudolf Habelt, 2015.

GORI, KRAPF 2015

Maja Gori, Tobias Krapf, « The Bronze and Iron Age pottery from Sovjan », Iliria 39, 2015, p. 91-135.

KRAPF $2016 a$

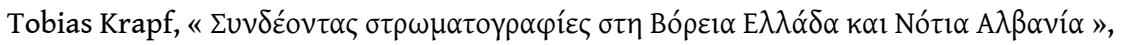

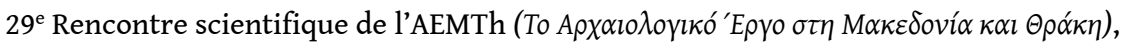
Thessalonique, 3-5 mars 2016.

KRAPF 2016b

Tobias Krapf, « From Central Greece to the North and then Westwards? Tracing Influences in Matt Painted Pottery from MBA to EIA », HESPEROS. The Aegean seen from the West, 16th International Aegean Conference, Ioannina, 18-21 mai 2016.

KRAPF 2016c

Tobias Krapf, « Balkan Bronze Age borderlands. Along ancient routes from the Aegean to Albania, 
FYROM, Kosovo and SW Bulgaria ", HESPEROS. The Aegean seen from the West, 16th International Aegean Conference, Ioannina, 18-21 mai 2016.

KRAPF et al. 2016

Tobias Krapf et al., « Southern Balkan regional variety and connectivity: Results of a new international collaboration ", 117th Annual Meeting of the American Archaeological Institute, San Francisco, 6-9 janvier 2016.

KORKUTI 1995

Muzafer Korkuti, Neolithikum und Chalkolithicum in Albanien, Mayence, P. von Zabern Verlag, 1995.

\section{NOTES}

1. Voir BCH 138 (2014), p. 795 ; BCH 139-140 (2015-2016).

2. BCH 135 (2011), p. 663-667.

3. KORKUTI 1995, p. 92-93.

4. GORI, KRAPF 2015.

5. $B C H$ 139-140 (2015-2016).

6. GORI 2015, p. 27.

7. KRAPF 2016a ; KRAPF $2016 b$.

8. Voir BCH 139-140(2015-2016) ; KRAPF et al. 2016 ; KRAPF 2016 c.

9. La rencontre, intitulée «Balkan Bronze Age Borderland II, Rethinking Southern Balkan LBA / EIA Interaction » (19-24 juillet 2016) a eu lieu à la maison suédoise de Kavala (Grèce) et a réuni 14 archéologues de sept pays.

10. Voir supra, n. 2.

11. ASLAKSEN, GORI, KRAPF 2016. Un article sur ces premiers résultats a aussi été soumis à la revue STAR (Science and Technology of Archaeological Research).

12. Voir BCH 136-137 (2012-2013), p. 698-699 ; BCH 139-140 (2015-2016).

13. BCH 136-137 (2012-2013), p. 706.

14. $B C H 135$ (2011), p. 680-683.

15. BCH 136-137 (2012-2013), p. 720.

16. En particulier un trésor de 618 monnaies d'argent hellénistiques, GJONGECAJ 1985 et Coin Hoards VIII, 1994, n² 299.

17. La numérisation des archives photographiques de notre collègue $P$. Lera a permis de constater que, sur certains sites (Gradisht de Symize, Zaradisht, Bellovoda, Mborja...), les vestiges architecturaux se sont considérablement dégradés en l'espace de trente ans. On comprend mieux l'urgence de sauvegarder les données architecturales encore visibles en réalisant, dans un premier temps, des relevés photogrammétriques.

18. Ce travail a été effectué dans le cadre du mémoire de fin d'études à l'École supérieure des géomètres et topographes (ESGT) du Mans. 
INDEX

Année de l'opération : 2016

lieux https://ark.frantiq.fr/ark:/26678/pcrt19bSKWqKS7

sujets https://ark.frantiq.fr/ark:/26678/pcrtx4sFxmENjb, https://ark.frantiq.fr/ark:/26678/

pcrt1DMOWvDF4j, https://ark.frantiq.fr/ark:/26678/pcrtQuZiT5nYY2

Thèmes : EFA

\section{AUTEURS}

\section{PETRIKA LERA}

Université Fan S. Noli de Korçë, Institut archéologique de Tirana

\section{GILLES TOUCHAIS}

Université Paris 1, UMR 7041 « Archéologies et Sciences de l'Antiquité (ArScAn) »

\section{CÉCILE OBERWEILER}

UMR 7041 « Archéologies et Sciences de l'Antiquité (ArScAn)»

\section{OLE CHRISTIAN ASLAKSEN}

Université de Göteborg

CHARLOTTE BLEIN

EHESS, UMR 5189 « Histoire et Sources des Mondes Antiques (HiSoMA)»

ATHINA BOLETI

UMR 7041 « Archéologies et Sciences de l'Antiquité (ArScAn)»

\section{GAZMEND ELEZI}

University of California (UCLA)

\section{LIONEL FADIN}

École française d'Athènes

\section{MAJA GORI}

Université de Bochum

\section{TOBIAS KRAPF}

Université de Bâle, Université de Paris 1, École suisse d'archéologie en Grèce

\section{YANNIS MANIATIS}

Centre Demokritos

\section{CHARLOTTE ODIE}

École supérieure d'ingénieurs géomètres et topographes 